\title{
Study on Influence of Different Gamma-ray Energy on Images of an Industrial CT System
}

\author{
Hanafi Ithnin ${ }^{1,2}$, Elmy Johanna Mohamad ${ }^{1, *}$, Nazrul Hizam Yusoff ${ }^{2}$, Hearie \\ Hassan $^{2}$, Norliana Mohd Lip ${ }^{3}$
}

\author{
${ }^{1}$ Instrumentation and Sensing Technology Research Group (InSet), Faculty of Electrical and Electronic Engineering, \\ University Tun Hussein Onn, Parit Raja 86400, Malaysia. \\ ${ }^{2}$ Industrial Technology Division, Malaysian Nuclear Agency, MOSTI, Kajang 43000, Malaysia. \\ ${ }^{3}$ Faculty of Computer and Mathematical Science, Universiti Teknologi Mara, Seremban 70300, Malaysia.
}

Received 16 November 2017; accepted 6 June 2018, available online 6 August 2018

\begin{abstract}
Gamma transmission computed tomography is a powerful imaging techniques that provide a crosssectional view or a volumetric view of the interior of an object. Therefore, reveal structural information of an object as if it had been cut open for viewing. By utilizing the properties of gamma-ray attenuation, images were reconstructed from photon counting data by gamma-ray sensor. Information revealed on these images depends greatly on resolution of the reconstructed images. In this study, the effect of gamma-ray energy used on a portable CT images is studied. Lab sample of four different material densities were used with three different gamma-ray sources namely, Am-241, Ba-133 and Cs-137 to study the effect of energy on the reconstructed images. The best reconstructed image that describes the structure of the sample used in this study is from Ba-133 with energy of 356 $\mathrm{keV}$. The results provide information on suitability of the energy used for the range of material density use in this study.
\end{abstract}

Keywords: Gamma-ray CT, Computed Tomography, Image Reconstruction

\section{Introduction}

Radiation based computed tomography such as X-ray and gamma-ray has been known as a powerful technique for studying the inner structure of an opaque sample. Due to high penetration power, for gamma-ray especially and attenuation properties of these radiation, wide range of material can be imaged by these radiations. Although for a specific sample, optimum energy needs to be selected so the image reconstructed can distinguish between a small density differences. In medical CT energy used in a CT scan has been optimized mainly to reduce the dose for the patient [1]. For industrial CT the energy depends on the problems on hand and do not have a dose limitation on sample. Due to this factor, large number of application for radiation based industrial CT system is being used and rapidly increasing [2-3].

For an X-ray CT, variation of energy used can be adjusted to meet the attenuation requirement for the sample. However, this is not applicable for portable gamma CT system, whereas the energy depends on type of sealed source used. Thus study on suitability of energy used for the interested range of material density is needed. Three different gamma sources with different energy were used to obtain the ideal or right energy range for the interested sample. Hence, with the right range of energy, it will optimize the capability of the CT system so that the right information can be extract from the crosssection images. Furthermore, it is well known that different gamma-ray energy attenuated differently with different density of material. Therefore, in this study, four different materials were used, i.e. wood block, sand, water, and aluminum rod as shown in Figure 1. Density ranges of these materials are $0.5 \mathrm{~g} \mathrm{~cm}^{-3}$ and $2.7 \mathrm{~g} \mathrm{~cm}^{-3}$.

\section{Methodology}

Four different density materials (samples) were used in this study (wood block, water, sand and aluminum rod). Arrangement of the samples is illustrated as in Figure 1. These samples were arranged together at the center of the portable CT scanner. Measurements were made for these entire samples with three different gamma-ray sources. The gamma-ray sources used are Americium-241, (Am-241) Barium-133, (Ba-133) and Cesium-137, (Cs-137) which have $60 \mathrm{keV}, 356 \mathrm{keV}$ and $662 \mathrm{keV}$ photon energies respectively. The portable CT system used is based on first generation CT system which has one collimated line source and one gamma-ray sensor as shown in Figure 2 [4]. The source and sensors will move step by step of 2.5 millimeters in linear translation and parallel beam measurement will be taken along with the movement. After translational movement finished, the $\mathrm{C}$-frame will rotate for an angle of 5 degree and new parallel beam measurement is taken. This process will be repeated until 180 degree which gives overall 37 rotational steps. 


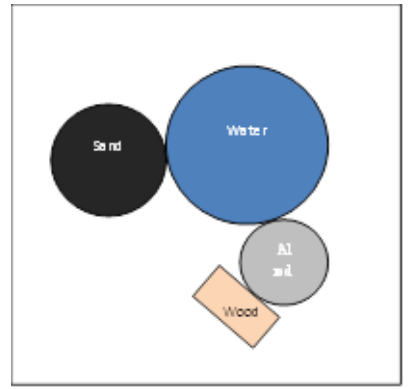

Fig. 1 Arrangement of the samples (Sand, Water, Aluminum rod and Wood block).

All movement is made by two stepper motors which controlling linear translation and rotation motion. The gamma-ray sensor on the system is connected to a single channel analyzer (SCA) which will gives the detected number of photon in a digital input. The data from SCA counting is arranged so that into an output file and later will be used as input for images reconstruction.

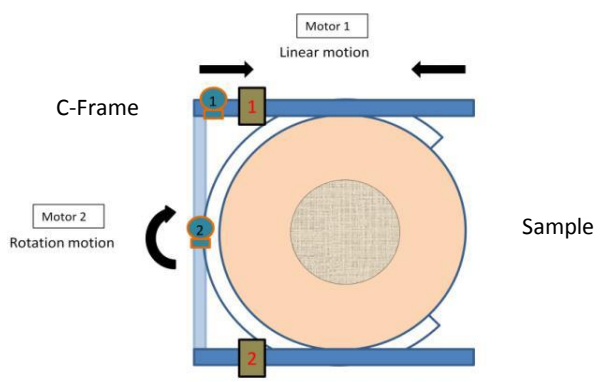

Fig. 2 Scanning system of portable gamma CT.

All movement is made by two stepper motors which controlling linear translation and rotation motion. The gamma-ray sensor on the system is connected to a single channel analyzer (SCA) which will gives the detected number of photon in a digital input. The data from SCA counting is arranged so that into an output file and later will be used as input for images reconstruction.

After the scanning process is finished, the data will be converted to a sonogram. Next the data will be filtered to reduce the background noise. In this study, simple filter back projection is used for image reconstruction process. All the images were reconstructed into a gray scale images and the analysis is using the gray scale value. The images from the three scanned is later analyzed using FIJI software which is a variation of ImageJ software [5].

\section{Results and Discussion}

This study is concentrated on determining effect of gamma ray energy used on a Portable CT and suitability of the energy used for ranges of material density. In order to get adequate images and at the same time optimizing the capability of the CT system so that the right information can be extract from the cross-section images, the influence of gamma energy and material density is investigated. Figure 3 shows the reconstructed images of the samples with Figure 3(b) from Barium-133 source which have the intermediate energy between the three sources, shows clear shape and elevation from the background. For the Amerecium-241 (Figure 3(a)), the cross section image of the rectangle wood sample is very poor. Unlike images from Figure 3 (a) \& (b), image for Cs-137 (Figure 3(c)) shows blurring on the edge of each sample.

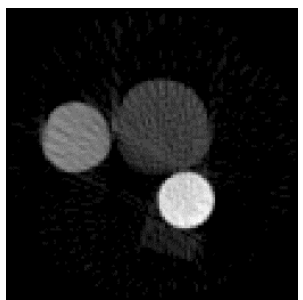

(a)

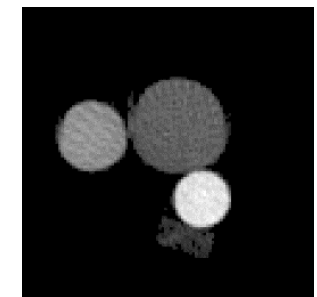

(b)

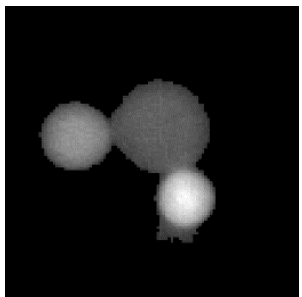

(c)

Fig. 3 Images reconstructed for three different gamma-ray source; a) Am-241 b) Ba-133 c) Cs-137

Figure 4 shows the histogram of the gray value from the three reconstructed images. The mean of these three gray values are $19.05,22.45$ and 18.61 for a, b and c respectively. This confirms image of Barium had more elevation of brighter region as seen. It is expected histogram of the gray value should have four peaks at different value to represent the four different density of the sample. The lowest peak should represent rectangle wood block which have the lowest gray value, second water circle, third sand and lastly the Aluminum rod which is the brighter in each image. From the distribution of the gray value it can be seen for image from Barium133 scan has showing clearly three peaks and the lower peak represent the wood is disperse at the lower region. Distribution of gray value from Americium-241 is high on the lower peak but that did not represent the wood block only. From the image it can be seen these dark pixel is the cumulative of the dark pixel of the wood block and noise or artifact at the background of the image. For Cesium-137 scanned image the distribution of gray value is broad at middle value as these values result from darkening edge of each sample shape.

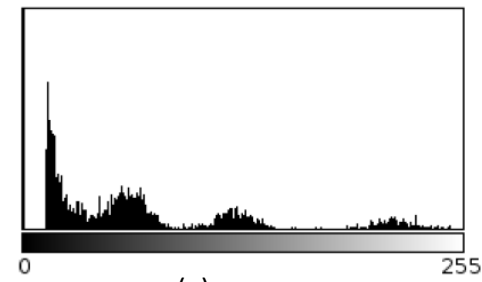

(a) 


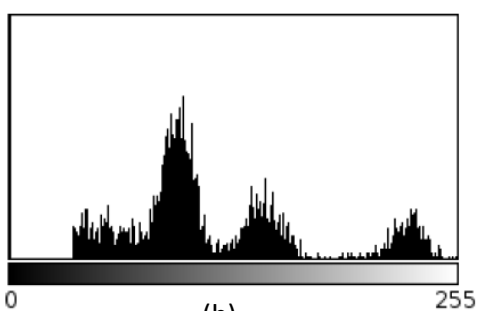

(b)

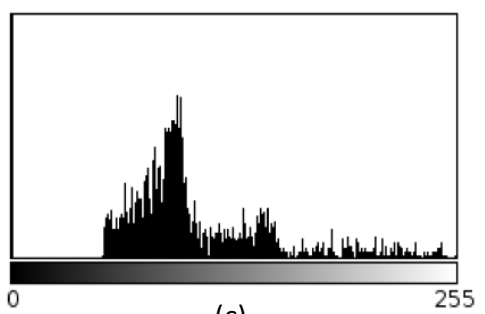

(c)

Fig. 4 Histogram of gray scale value for the three reconstructed images; a) Am-241 b) Ba-133 c) Cs-137

\section{Summary}

Based on the reconstructed images and histogram of the three images, it can be concluded and it is confirmed, Ba-133 which energy is $356 \mathrm{keV}$ that has the intermediate energy value between these three gamma-ray sources is the most suitable energy range for material density of the sample. For lower energy of Am-241 the overall image is darken and have many artifacts at the background. On the other hand for the high energy of Cs-137, the edge of each sample shape is blurred. This is due to at the high energy of Cs-137, $662 \mathrm{keV}$ the attenuation of the gammaray very poor at the edge hence there big difference of photon count at the center of each sample and edge of each sample. The result has given important information on what is the best gamma sealed source to be used for portable gamma-ray CT scanning of sample in the density range of $0.5 \mathrm{~g} \mathrm{~cm}^{-3}$ to $2.7 \mathrm{~g} \mathrm{~cm}^{-3}$.

\section{Acknowledgement}

The authors would like to extend their appreciation to all personnel of the Plant Assessment Technology group, Industrial Technology Division, Malaysian Nuclear Agency and Faculty of Electrical and Electronic Engineering, University Tun Hussein Onn (UTHM). The authors also would like to thanks all personnel that involve directly or indirectly in completing this study.

\section{References}

[1] D. J. Brenner, and E. J. Hall, N. Engl J Med, 357:2277-2284, (2007).

[2] L. De Chiffre, S. Carmignato, J.-P. Kruth, R. Schmitt, A. Weckenmann, Industrial applications of computed tomography, CIRP Annals Manufacturing Technology 63; 655-677, (2014)

[3] S.A. Legoupil, R. Maad, J. Nowakowski, D. Sankowski, J. Thyn, et.al, Industrial Process Tomography, IAEA-TECDOC-1589, IAEA, Vienna, (2008).

[4] J. Abdullah, H. Hassan, M. R. Shari, M. M. Ibrahim, N. Yussup, H. Ithnin, A. A. Mahmood, Developement and implementation of a portable nucleonic computed tomography system with clampon-features for engineering inspection, Jurnal Teknologi (Sciences \& Engineering) 77:17, 121-127, (2015).

[5] J. Schindelin, I. Arganda-Carreras, \& E. Frise, et al. "Fiji: an open-source platform for biological-image analysis", Nature methods 9(7): 676-682 (2012). 